\title{
Corrigendum: VEGF modulates erythropoiesis through regulation of adult hepatic erythropoietin synthesis
}

Betty YFY Tam, Kevin Wei, John S Rudge, Jana Hoffman, Joceyln Holash, Sang-ki Park, Jenny Yuan, Colleen Hefner, Cecile Chartier, Jeng-Shin Lee, Shelly Jiang, Nihar R Nayak, Frans A Kuypers, Lisa Ma, Uma Sundram, Grace Wu, Joseph A Garcia, Stanley L Schrier, Jacquelyn J Maher, Randall S Johnson, George D Yancopoulos, Richard C Mulligan \& Calvin J Kuo Nat. Med. 12, 793-800 (2006); published online 25 June 2006; corrected after print 6 April 2009

In the version of this article initially published, the name of one of the authors, Nihar R. Nayak, was misspelled as Nihar R. Niyak. The error has been corrected in the HTML and PDF versions of the article.

\section{Corrigendum: Bone marrow stromal cells attenuate sepsis via prostaglandin $\mathrm{E}_{2}$-dependent reprogramming of host macrophages to increase their interleukin-10 production}

\begin{abstract}
Krisztián Németh, Asada Leelahavanichkul, Peter S T Yuen, Balázs Mayer, Alissa Parmelee, Kent Doi, Pamela G Robey, Kantima Leelahavanichkul, Beverly H Koller, Jared M Brown, Xuzhen Hu, Ivett Jelinek, Robert A Star \& Éva Mezey Nat. Med. 15, 42-49 (2009); published online 21 November 2008; corrected after print 6 April 2009
\end{abstract}

In the version of this article initially published, the labeling in (Figure 4) was incorrect. In panel (b), the cells in the left two FACS plots are shown based on their size (FSC, $y$ axis) and CD11b expression ( $x$ axis), and the cells in the right two FACS plots are shown based on their F4/80 expression ( $y$ axis) and GR1 expression ( $x$ axis). In panel (c), the curves should start at $1 \mathrm{~h}$. In panel (d), the text labeling the $y$ axis should read "in vitro," not "in vivo." The errors have been corrected in the HTML and PDF versions of this article.

\section{Corrigendum: The cerebral cavernous malformation signaling pathway promotes vascular integrity via Rho GTPases}

Kevin J Whitehead, Aubrey C Chan, Sutip Navankasattusas, Wonshill Koh, Nyall R London, Jing Ling, Anne H Mayo, Stavros G Drakos, Christopher A Jones, Weiquan Zhu, Douglas A Marchuk, George E Davis \& Dean Y Li

Nat. Med. 15, 177-184 (2009); published online 18 January; corrected after print 6 April 2009

In the version of this article initially published, Christopher A. Jones and Weiquan Zhu were not included in the list of authors. The error has been corrected in the HTML and PDF versions of the article.

Corrigendum: Effector memory T cell responses are associated with protection of rhesus monkeys from mucosal simian immunodeficiency virus challenge.

Scott G Hansen, Cassandra Vieville, Nathan Whizin, Lia Coyne-Johnson, Don C Siess, Derek D Drummond, Alfred W Legasse, Michael K Axthelm, Kelli Oswald, Charles M Trubey, Michael Piatak Jr, Jeffrey D Lifson, Jay A Nelson, Michael A Jarvis \& Louis J Picker Nat. Med. 15, 293-299 (2009); published online 15 February 2009; corrected after print 6 April 2009

In the version of this article initially published, a "left" and "right" designation was switched in the legend for Figure 4d. The legend should read "FCICA of peripheral blood CD8 ${ }^{+} \mathrm{T}$ cells from the four protected vaccinees, examining the response of these cells to SIV proteins that were (RevTat-Nef) or were not (Pol and Vif) expressed by the administered RhCMV vectors before (left) and $133 \mathrm{~d}$ after (right) initiation of the SIVmac239 intrarectal challenge protocol." The error has been corrected in the HTML and PDF versions of the article.

\section{Erratum: Straight talk with...Mac Cowell and Jason Bobe}

Prashant Nair

Nat. Med. 15, 230-231 (2009); published online 5 March 2009; corrected after print 6 April 2009

In the print version of this interview, the first response contained an unrelated excerpt from the previous month's Q \& A. The text begins with "who could mobilize..." and ends with "...results and more results." The error did not appear online in the HTML and PDF versions of the article. 